\title{
The Change of Grip Strength in a Patient with Congenital Myotonic Dystrophy Over a 4-year Period
}

\author{
Shin KIKUCHI ${ }^{1}$, Naoki KozUKA ${ }^{2}$, Eiji UCHIDA ${ }^{3}$, Takafumi NiNOMIYA ${ }^{1}$, \\ Haruyuki TATSUMI ${ }^{1}$, Hidekatsu TAKEDA ${ }^{2}$ and Nobutada TACHI ${ }^{2}$ \\ ${ }^{1}$ Department of Anatomy 1, Sapporo Medical University School of Medicine, South 1, West 17, Chuo-ku, Sapporo 060-8556, Japan \\ ${ }^{2}$ School of Health Sciences, Sapporo Medical University, South 1, West 17, Chuo-ku, Sapporo 060-8556, Japan \\ ${ }^{3}$ Department of Human Science, Faculty of Human Studies, Taisho University, 3-20-1 Nishisugamo, Toshima-ku, Tokyo \\ 170-847, Japan
}

\begin{abstract}
Myotonic dystrophy (MyD) is a neuromuscular disease that is autosomal dominant and the most common form of muscular dystrophy affecting adults. The clinical features of MyD include a multisystemic disorder characterized by myotonia, progressive muscle weakness and wasting, cataracts, premature balding and mental retardation. The most severe type of MyD is classified as congenital MyD (CMyD). The muscle weakness in CMyD is very severe, but muscle development can be observed in the period of growth. However, no clinical case of this type has been reported yet. Therefore, we report on a girl with CMyD who had an increase in muscle strength over a four-year period. The girl with CMyD participated in this study from the age of 9 to the age of 12. The measurement of muscle strength was recorded as the maximum score of grip strength with the use of dynamometers. Grip strength was assessed once a year by the same two physical therapists. Grip strength of CMyD for each year was markedly weak when compared with the normal controls, but muscle strength changed within some specific growth areas. The muscle weakness in CMyD was remarkable, but the result showed that specific muscle strength of CMyD in childhood was actually increased.
\end{abstract}

Key words: congenital myotonic dystrophy, development, grip strength

(J Jpn Phys Ther Assoc 11: 23-27, 2008)

$\mathbf{M}$ yotonic dystrophy (MyD) is an autosomal dominant neuromuscular disease caused by amplification of an unstable CTG repeat in the 3' untranslated region on human chromosome $19 \mathrm{q} 13.3^{1)}$. MyD has an incidence of 5.5 in 100,000 and is the most common form of adult-type muscular dystrophy ${ }^{2}$. The clinical features of MyD are markedly variable, characterized by myotonia, progressive muscle weakness and atrophy, cataracts, premature balding and mental retardation ${ }^{3)}$. The clinical severity shows extreme variation even among family members. The symptoms and severity of $\mathrm{MyD}$ are related to age at the time of onset. The most mild and late-onset form of MyD is classified as adult type myotonic dystrophy (AMyD). On

Received: June 2, 2006

Accepted: January 28, 2008

Correspondence to: Shin kikuchi, Department of Anatomy 1, Sapporo Medical University School of Medicine, South 1, West 17, Chuo-ku, Sapporo 060-8556, Japan

e-mail: ksin@sapmed.ac.jp the other hand, the type that is most severe, with onset from birth is congenital myotonic dystrophy (CMyD) ${ }^{4}$. Progressive muscle weakness is one of the cardinal symptoms in MyD but, in CMyD, it has been suggested that those who survive CMyD for a year after birth have an improvement in their muscle strength ${ }^{5)}$. However, there is no detailed report in the literature about muscle strength improvement in CMyD.

The main symptoms of MyD are progressive muscle weakness and atrophy. The degree of muscle weakness and the severity of other clinical symptoms of MyD are correlated with the length of the CTG repeat ${ }^{6-10)}$. Gharehbaghi-Schnell et al. indicated a correlation between clinical severity and the CTG repeat based on the results of comparing the CTG repeat and the Muscular Disability Rating Scale (MDRS) scores of 18 patients with $\mathrm{MyD}^{8)}$. Eguchi et al. reported a correlation between the number of CTG repeats and three clinical features (age at the time of onset, degree of mental retardation and severity of muscle 
weakness), in 35 individuals with MyD. These results showed good correlations between the number of CTG repeats and these three items ${ }^{9}$. Kozuka et al. measured muscle strength of seven muscles and showed that grip strength tended to decrease inversely in relation to the size of the CTG repeat ${ }^{10)}$. In addition, this result suggested that grip strength might be one marker to evaluate the degree of clinical severity ${ }^{9}$. In fact, grip strength is used as a tool for measurement of the muscle strength in $\mathrm{MyD}^{11-19}$. We searched for articles using the term grip strength as a tool in MyD in PubMed using the terms "myotonic dystrophy" and "muscle strength". We excluded non-English literature and did not consider quantitative muscle strength such as manual muscle testing (MMT). The search yielded 17 articles, of which 9 were the best matches available. All 9 articles measured grip strength as a parameter. Information was extracted from the articles on (1) the number of patients, (2) gender and mean age, (3) the number of CTG repeats, (4) methods used for measurement and (5) mean grip strength. Our review showed that there was no article which measured the age-related changes of grip strength in MyD (Table 1). Nor was there any article evaluating the CMyD (the youngest participant was 8 years old but the category of CMyD was not used $\left.{ }^{12)}\right)^{11-19}$. It is important for physical therapists to recognize whether muscle strength can increase in patients with CMyD, but there have been no reports investigating the change of muscle strength quantitatively in CMyD.

We encountered one patient with $\mathrm{CMyD}$ whose grip strength increased over four years. The aims of this report, through this one case, were to show the change specificity of grip muscle strength in $\mathrm{CMyD}$ as a basic factor for prognosis and to design an effective physical therapy program for CMyD.

\section{Case}

\section{Progress before evaluation of muscle strength}

A female child, diagnosed with $\mathrm{CMyD}$, participated in this study. Her CTG repeat size was about 2,200 repeats ${ }^{10)}$. The subject's growth was obtained from her clinical chart and the data collection was continued until she was 8 years old.

History of subject from birth to 2 months: She was born at 35 weeks of gestation after a cesarean section was performed due to polyhydramnios and fetal asphyxia; her birth weight was 2,238 grams. Her Apgar scores were 1 and 3 at 1 and 5 minutes respectively. She had general muscle hypotonia (frog leg position) and required ventilation for one month after birth. At 2 months, she was discharged from the hospital because she could sufficiently swallow and suck.

Milestones assessment: Tracking, 4 months; Moro reflex, 4 months; smile, 5 months; handkerchief on face, 6 months; rolling over, 10 months; crawl, 12 months; standing and walking with support, 16 months; walking alone (about 10 steps), 22 months; running, 3 years; speech (one word), 3 years 9 months; climbing up and down the stairs, 3 years 9 months; pedaling a bicycle with support, 4 years 1 month; express the need to urinate, 4 years 1 month; speech (over 3 words), 7 years; jumping, 7 years; speech (making a sentence), 8 years.

The subject had never done any specific muscle strengthening training or physical therapeutic exercise until the time of this study. The measurements were done in August every year from 2001 to 2004, from the age of 9 to 12 years. She did not undertake any specific physical therapy or muscle training. Her lifestyle showed no difference until she reached 12 years of age. Her mother was diagnosed with AMyD with no symptoms, except for grip myotonia. The mother had no problem with ADL.

Before any measurement of muscle strength was done, the normal range of motion was verified and no deformity was found. Hyper- or hypomobility was not observed in the screening test for ROM in the subject. At the ages of 9 to 12 , she was $121.0,125.1,128.2$ and $133.4 \mathrm{~cm}$ tall and weighed 21.2, 22.0, 26.0 and $26.2 \mathrm{~kg}$, respectively.

The subject and her mother were informed about the parameters of this study and consented to a series of investigations. In addition, this study obtained approval from the Committee for Graduate School of Health Sciences, Sapporo Medical University (approval number: 2001MP-02-12).

\section{Methods}

\section{Measurement protocol}

Grip strength tends to decrease in inverse relation to the size of the CTG repeats. The grip strength was measured by the same handgrip dynamometer (TK-1201, TAKEI KK) each year. The grip strength test was performed by the same two physical therapists and was measured in accordance with a standard protocol. Grip strength was measured 3 times for each hand (total 6 times in all), each separated by a $30 \mathrm{sec}$ rest period. The highest result of the six trials was used as the grip strength. The results were compared with the normal strength that was matched by age $(9,10,11$ and 12 years old) and gender from the investigation of physical strength and activity in the 2002 fiscal year ${ }^{20)}$.

Intraclass correlation coefficients (ICC) were used to analyze the reliability of each measurement using SPSS Ver13.0J for Windows. Analysis of reliability for grip strength data was conducted for the 3 measurement values for each hand (right and left hand) for each year (4 years).

\section{Validity of the instrument}

The validity of the handgrip dynamometer was measured with Fess's method each year for the entire four- 


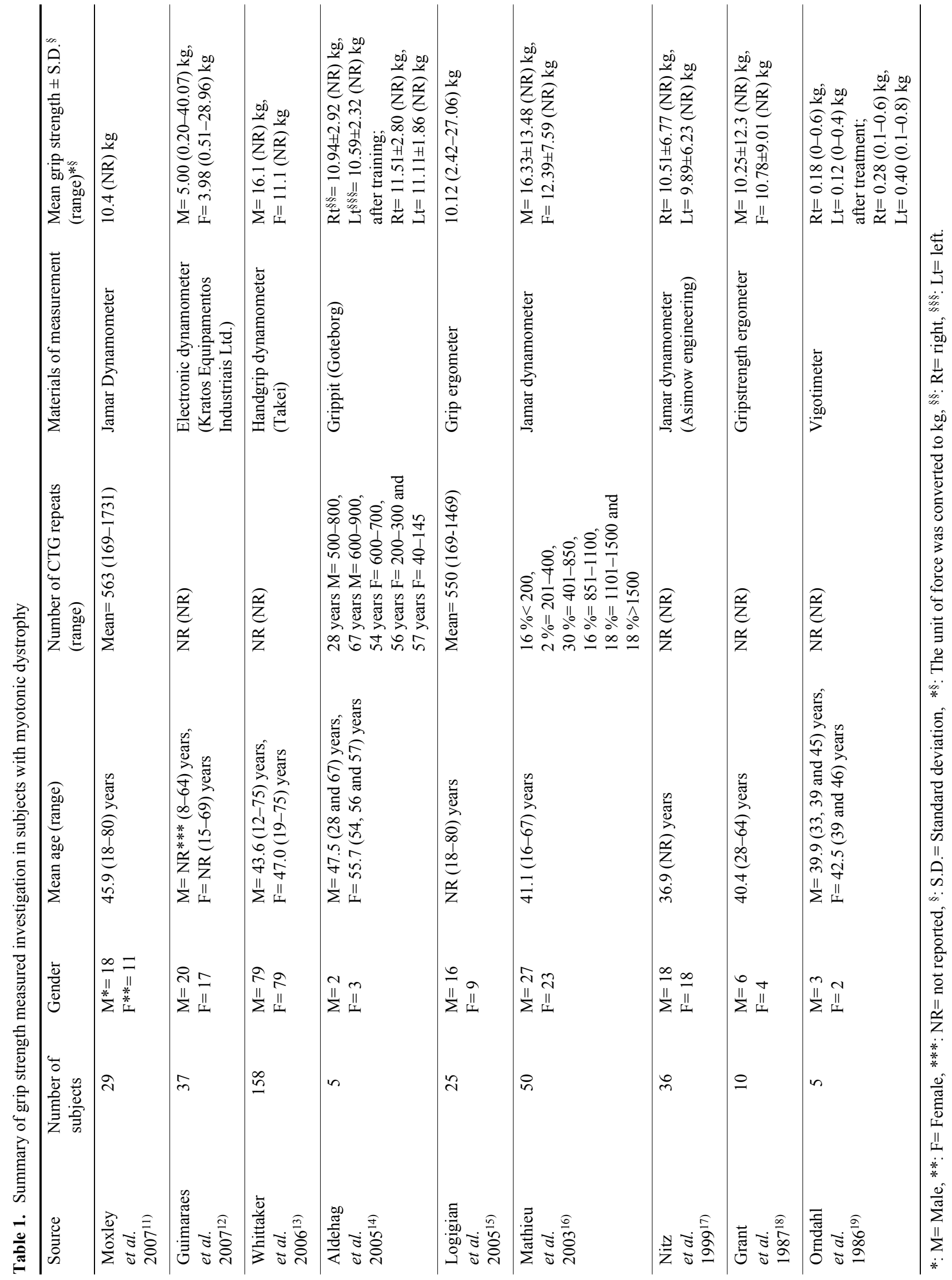


Table 2. Height, weight and grip strength and percentiles of normal controls in each year

\begin{tabular}{|c|c|c|c|c|}
\hline & $9 y^{*}$ & $10 \mathrm{y}$ & $11 \mathrm{y}$ & $12 \mathrm{y}$ \\
\hline \multicolumn{5}{|l|}{ Height $(\mathrm{cm})$} \\
\hline subject & 121.0 & 125.1 & 128.2 & 133.4 \\
\hline N.C. $* *(\text { mean } \pm \text { S.D. })^{* * *}$ & $133.8 \pm 6.3$ & $140.6 \pm 6.9$ & $147.4 \pm 6.6$ & $152.3 \pm 5.6$ \\
\hline$\%$ & 90.4 & 89.0 & 87.0 & 87.6 \\
\hline \multicolumn{5}{|l|}{ Weight $(\mathrm{kg})$} \\
\hline subject & 21.2 & 22.0 & 26.0 & 26.2 \\
\hline N.C. $($ mean \pm S.D. $)$ & $30.1 \pm 5.4$ & $34.7 \pm 6.9$ & $39.9 \pm 7.6$ & $44.7 \pm 7.5$ \\
\hline$\%$ & 70.5 & 63.3 & 65.1 & 58.7 \\
\hline \multicolumn{5}{|l|}{ Grip strength (kg) } \\
\hline subject (maximum) & 6.5 & 5.5 & 7.5 & 7.5 \\
\hline N.C. $($ mean \pm S.D. $)$ & $14.2 \pm 3.3$ & $16.8 \pm 3.9$ & $20.0 \pm 4.5$ & $22.3 \pm 4.4$ \\
\hline$\%$ & 45.8 & 32.7 & 37.5 & 33.6 \\
\hline
\end{tabular}

year period $^{21)}$. The handgrip dynamometer was in error by less than $0.75 \mathrm{~kg}$.

\section{Results}

\section{Grip strength}

The ICC score was 0.83 , indicating that the results were very reliable.

As shown in Table 2, the grip strength results of the subject by year were $6.5 \mathrm{~kg}$ (Left [L]: $5.5-6.0 \mathrm{~kg}$, Right [R]: 6-6.5 kg) (minimum-maximum), $5.5 \mathrm{~kg}$ (L: 5-5.5, R: 4.5$5.0 \mathrm{~kg}), 7.5 \mathrm{~kg}$ (L: $6.0-7.0 \mathrm{~kg}, \mathrm{R}: 6.5-7.5 \mathrm{~kg}$ ) and $7.5 \mathrm{~kg}$ (L: 6-7.5 kg, R: 6-7.5 kg). The grip strength percentages were $45.8,32.7,37.5$ and $33.6 \%$ for the ages of $9,10,11$ and 12 , respectively, compared with normal control matched subjects by age and gender. The grip strength showed a moderate increase with aging, but not linearly. The difference between grip strength in this case and the normal control became larger year by year during the measurement over the four-year period.

\section{The changes of weight and height}

The height and weight values increased year by year (Table 2). However, the percentiles of the subject's height were below the normal average and decreased year by year $(90.4,89.0,87.0$ and $87.6 \%)$, but not to under $85 \%$. Percentiles of her weight were not linearly plotted (70.5, $63.3,65.1$ and $58.7 \%$ ), and the change pattern of weight was similar to that for grip-strength changes.

\section{Discussion}

Mathieu et al. reported that muscle strength tended to decrease with age in $\mathrm{MyD}^{16)}$. Eguchi et al., however, showed that the degree of muscle weakness varied in each case of AMyD, and there was little agreement on the muscle weakness found in $\mathrm{MyD}^{9}$ ). Until now it has been considered that high-resistance strength training causes overwork weakness in MyD. However, Tollback et al. demonstrated that patients with MyD had improved muscle strength without any observed negative side effect after highresistance training ${ }^{7)}$. Recently, Lideman et al. substantiated that strength training improved muscle function of patients with slowly progressive hereditary neuromuscular disorders $^{22)}$ such as CMyD. However, most of the subjects of these studies did not actually have CMyD, but rather AMyD, and there are actually very few studies on the changes of muscle strength with aging in CMyD. Therefore, we studied muscle strength changes in a patient with CMyD from the age of 9 to 12 years old.

The results of this study showed that the grip strength at 12 years of age was increased in comparison with that at 9 years of age. This result might show the possibility of increased muscle strength with development in CMyD. The development rate of muscle strength in CMyD was very slow in comparison with the normal control. The grip strength in this case was $50 \%$ when compared with the normal control, and the gap tended to increase year by year. In addition, the muscle strength did not linearly increase. The pattern of percentile change showed the similarity between grip strength and weight. In particular, the measured values of grip strength and weight decreased from 9 years old to 10 years old. Weight values are affected by many elements and a decrease of weight in childhood usually suggests an abnormal condition or a nutritional problem. The cause of the weight decrease was not known in this case, but the decrease of grip strength from ages 9 to 10 might have been connected with not only pathological 
effects, but also the total body condition. Therefore, the decrease of grip strength from 9 to 10 in this particular case is not necessary by the negative evidence for an increase of muscle strength in patients with myotonic dystrophy.

In the skeletal muscle immature fibers are observed predominantly in childhood in patients with $\mathrm{CMyD}^{23)}$, but in adults with CMyD pathological observations are similar to those of late onset $\mathrm{MyD}^{24)}$. Kikuchi and colleagues reported that the muscle pathological observation of CMyD with growth to 10 years old was similar to that of adult onset MyD and type IIC (immature) fibers were not detected $^{25)}$. These reports suggest that the maturation of the skeletal muscle fiber type in CMyD progresses with aging, but that for muscle the pathological observations are the same as for AMyD. Therefore, in this case, muscle strength increased but the muscle expanded in a different manner from the normal control.

This case, suggests that CMyD patients have the possibility to increase muscle strength from 9 to 12 years of age, and Tollback et al. reported that muscle strength was improved without any negative side effect after highresistance training ${ }^{7)}$. For physical therapy in CMyD, our results and the previous report suggest a need for physical exercise including a high-resistance training program to limit the growing gap between children with CMyD and normal children, after first confirming the total body development and condition.

\section{Conclusion}

The importance of this study is that the change of muscle strength in CMyD showed an unstable increase and the difference of muscle strength between the patient with CMyD and the normal control became greater within the period of 9 to 12 years of age. It is difficult to arrive at a general conclusion as to the change of muscle strength in CMyD, because there was only one participant in this study. Therefore, we think it is worthwhile to expand this research with a greater number of similar subjects.

\section{References}

1) Kozuka N, Tachi N, et al.: Non-RI PCR southern method for analysis of CTG repeat in myotonic dystrophy. No to Hattatsu 35: 380-387, 2003 (in Japanese).

2) Osame M, Furusho T: Genetic epidemiology of myotonic dystrophy in Kagoshima and Okinawa districts in Japan. Clin Neurol 23: 1067-1071, 1983 (in Japanese).

3) Harper PS: Myotonic Dystrophy. 3rd ed, WB Saunders, 2001.

4) Harper PS: Congenital myotonic dystrophy in Britain. I. Clinical aspects. Arch Dis Child 50: 505-513, 1975.

5) Reardon W, Newcombe R, et al:: The natural history of congenital myotonic dystrophy: mortality and long term clinical aspects. Arch Dis Child 68: 177-181, 1993.

6) Dubowitz V, Heckmatt J: Management of muscular dystrophy. Pharmacological and physical aspects. Br Med Bull 36: 139-
144, 1980.

7) Tollback A, Enriksson S, et al.: Effects of high resistance training in patients with myotonic dystrophy. Scand J Rehab Med 31: 9-16, 1999.

8) Gharehbaghi-Schnell EB, Finsterer J, et al:: Genotypephenotype correlation in myotonic dystrophy. Clin Genet 53: 20-26, 1998.

9) Eguchi I, Koike R, et al:: Correlation between degrees of the CTG repeat expansion and clinical features of myotonic dystrophy. Clin Neurol 34: 118-123, 1994 (in Japanese).

10) Kozuka N, Tachi N, et al.: Molecular and clinical studies in myotonic dystrophy - the relation between trinucleotide (CTG) repeat and muscle strength - . The Society for the Study of Medical Basis of Physical Therapy 6: 9-15, 2002 (in Japanese).

11) Moxley RT 3rd, Logigian EL, et al.: Computerized hand grip myometry reliably measures myotonia and muscle strength in myotonic dystrophy (DM1). Muscle Nerve 36: 320-328, 2007.

12) Guimaraes AS, Carlosson GE, et al.: Bite force and handgrip force in patients with molecular diagnosis of myotonic dystrophy. J Oral Rehabil 34: 195-200, 2007.

13) Whittaker RG, Ferenczi E, et al:: Myotonic dystrophy: practical issues relating to assessment of strength. J Neurol Neurosurg Psychiatry 77: 1282-1283, 2006.

14) Aldehag AS, Jonsson $\mathrm{H}$, et al.: Effects of a hand training programme in five patients with myotonic dystrophy type 1 . Occup Ther Int 12: 14-27, 2005.

15) Logigian EL, Blood CL, et al:: Quantitative analysis of the "warm-up" phenomenon in myotonic dystrophy type 1 . Muscle Nerve 32: 35-42, 2005.

16) Mathieu J, Boivin $\mathrm{H}$, et al.: Quantitative motor assessment in myotonic dystrophy. Can J Neurol Sci 30: 129-136, 2003.

17) Nitz JC, Burns YR, et al:: A longitudinal physical profile assessment of skeletal muscle manifestations in myotonic dystrophy: Clin Rehabil 13: 64-73, 1999 .

18) Grant R, Sutton DL, et al:: Nifedipine in the treatment of myotonia in myotonic dystrophy. J Neurol Neurosurg Psychiatry 50: 199-206, 1987.

19) Orndahl G, Sellden U, et al:: Myotonic dystrophy treated with selenium and vitamin E. Acta Med Scand 219: 407-414, 1986.

20) Ministry of Education, Culture, Sports, Science and Technology: The investigation of physical strength and activity in Heisei 14 fiscal year. http://www.mext.go.jp/ b_menu/toukei/001/022/2002.htm (in Japanese).

21) Fess EE: A method for checking Jamar dynamometer calibration. J Hand Ther 1: 28-32, 1987.

22) Lindeman E, Spaans S, et al.: Progressive resistance training in neuromuscular patients. Effects on force and surface EMG. J Electromyogr Kinesiol 9: 379-84, 1999.

23) Farkas-Bargeton E, Barbet JP, et al.: Immaturity of muscle fibers in the congenital form of myotonic dystrophy: its consequences and its origin. J Neurol Sci 83: 145-159, 1988.

24) Tanabe Y, Nonaka I: Congenital myotonic dystrophy. Changes in muscle pathology with ageing. J Neurol Sci 77: 5968, 1987.

25) Kikuchi S, Kozuka N, et al:: The relation between CTG triplet repeat size and muscle histopathological observation in congenital myotonic dystrophy. Sogo Reha 33: 167-173, 2005 (in Japanese). 